\title{
EVALUATION OF DERIVATIVE FREE KALMAN FILTER AND FUSION IN NON-LINEAR ESTIMATION
}

\author{
S. K. Kashyap \\ Scientist, Flight Mechanics and Control Division \\ National Aerospace Laboratories \\ Bangalore-560 017 \\ Email: sudesh@css.nal.res.in
}

\author{
J.R. Raol \\ Head, Flight Mechanics and ControlDivision \\ National Aerospace Laboratories \\ Bungalore-560017 \\ Email: irranl@ess.nal.res.in
}

\begin{abstract}
In recent literature a derivative free Kalman filter (DFKF) a method that propagates mean and covariance using nonlinear transfonmation is frequenly used. In this paper i) factorized version of EKF (UD Extended Kalman Filter or UDEKF) and ii) DFKF are studied and evaluated using various sets of simulated data of the non-linear systems. Sensinvity study of DFKF with respect to tuning parameters used in creation of sigma points and the associated weights is carried out. DFKF is more accurate and easier to implement. A data fusion scheme is evolved and presented based on DFKFfor similar sensors. Its performance is evaluated. It is observed that fusion enhances the estimation accuracy of the slate of non-linear plant. Application of DFKF to non-linear parameter estimation problem is also demonstrated.
\end{abstract}

Keywords: Non-linear systems. Target tracking. Kalman filtering, Derivative free transfonmation and Kalman filter, Data fusion.Parameter estimation

\section{Introduction}

\subsection{Derivative Free Transformation and Kalman Filter (DFKF)}

Fig. I shows pictorial representation of DFT [1]. Consider propagation of a random variablexof dimension $\mathrm{L}(\mathrm{L}=2)$ through a non-linear function $y=f(x)$. Assume that mean and covariance of sigma points, shown by black dots in left side of fig. 1, for random variable are $\bar{x}$ and $P_{x}$ respectively. These sigma points and their associated weights are deterministically created by the following equations:

$$
\begin{aligned}
& \left.\begin{array}{ll}
\chi_{0}=\bar{x} & \\
\chi_{i}=\bar{x}+\left(\sqrt{(L+\lambda) P_{x}}\right)_{i} & i=1, \ldots, L \\
\chi_{i}=\bar{x}-\left(\sqrt{(L+\lambda) P_{x}}\right)_{i-L} & i=L+1, \ldots, 2 L
\end{array}\right\} \\
& W_{0 m}=\frac{\lambda}{L+\lambda} \\
& W_{0 r e}=\frac{\lambda}{L+\lambda}+\left(1-\alpha^{2}+\beta\right) \\
& W_{i m}=W_{i m}=\frac{1}{2(L+\lambda)} \quad i=1, \ldots, 2 L
\end{aligned}
$$

Here, ' $m$ ' and ' $c$ ' stand for mean and covariance respectively. The scaling parameters used for the creation of sigma points and their associated weights are: $\alpha$ that determines the spread of sigma points around $\bar{x}, \beta$ is used to incorporate any prior knowledge about distribution of, $\lambda=\mathbf{a}^{2}(L+\kappa)-\mathrm{L}$ and $\boldsymbol{K}$ is the secondary tuning parameter. The sigma points created are propagated through the non-linear function $\left(y_{i}=f\left(\chi_{i}\right)\right.$, where, $i=0, \ldots, 2 L)$ resulting in transformed sigma points (black dots in right side of fig. 1). The mean and covariance of transformed points are formulated as:

$$
\begin{aligned}
& \bar{y}=\sum_{i=0}^{2 L} W_{i m} y_{i} \\
& P_{y}=\sum_{i=0}^{2 L} W_{i c}\left\{y_{i}-\bar{y}\right\}\left\{y_{i}-\bar{y}\right\}^{T}
\end{aligned}
$$

Subsequently the sigma-point computations for basic state estimation are given in details, $\mathrm{A}$ data fusion scheme for similar sensors using DFKF to extract more information about an entity of interest is then evolved based on the DFKF. Various aspects like: DF-DFKF initialization, augmented state and its error covariance, sigma-points computation, state and covariance propagation, and state and covariance update will be discussed and presented in details in the final paper. 


\section{Results and Discussions}

\subsection{Kinematics Consistency}

The performance of the filters for kinematics consistency using realistic longitudinal short period and lateral-directional data generated from a six-degree-of freedom simulation of an aircraft is evaluated. The basic kinematics required in state estimation is then presented by state and observation Models. The measurement noise with SNR of 10 is added only to the observables and no noise is added to the rates and accelerations during the data generation. The results shown in fig. 2 are generated for 25 Monte Carlo simulations. Fig. 2 shows the comparison of true, measured and estimated observables. From the plots it is clear that wherever (e.g. between $0-5$ seconds or around 10 second) the non-linearity in measurement data is more severe, the performance of UDEKF is degraded as compared to DFKF.

\subsection{Data Fusion}

Consider the vehicle reentry problem shown in fig. 3. It is assumed that a vehicle entering the atmosphere at high altitude and at high speed is tracked by two ground-stationed sensors with different accuracies. It is assumed that sensors are placed nearby. The measurements from either of sensors are $\mathbf{m}$ terms of range and bearing. The strong nonlinearities present in vehicle dynamic are due to the different types of forces acting on it The most dominant force is aerodynamic drag as a function of vehicle speed and altitude. Gravitational force accelerates the vehicle towards the center of Earth. In initial phase of flight, vehicle has almost ballistic trajectory but as density of the atmosphere increases, drag effects become more important and the vehicle rapidly decelerates until its motion is almost vertical. The initial state of vehicle is equal to $[6500.4,349.14,-1.8093,-6.7967,0.6932]$. The data is simulated for total number of $\mathrm{N}-1450$ scans. The vehicle is continuously tracked by two sensors in proximity at $\left(\mathrm{n},=6375 K m, y_{r}=\mathbf{0} \mathbf{K m}\right)$. The rate at which measurements arrive is at a frequency of $5 \mathrm{~Hz}$ i.e. sampling interval $\mathrm{T}=0.2$ seconds. It is assumed that first sensor gives good bearing information but has noisy range measurement and vice-versa for second sensor (thought this may not be true in general, it is assumed here for the sake of performance evaluation of the algorithm). The standard deviations of range and bearing noises used in simulation are: Sensor 1: $\sigma_{1 r}=1 \mathrm{Km}, \sigma_{1 \theta}=$ $0.05 \mathrm{deg}$, Sensor 2: $\sigma_{2 r}=0.22 \mathrm{Km}, \sigma_{2 \theta}=1 \mathrm{deg}$. The results are generated for 25 Monte Carlo simulations and performances of DF-DFKF and two DFKF (i.e. for sensor $1 \&$ sensor 2 respectively) are compared. It is clear from fig. 4 that fused state, as compared to estimated state from other two methods, is close to trie state. It is clear that data fusion increases the estimation accuracy that would not have been possible using single sensor measurements.

\subsection{Parameter Estimation}

DFKF algorithm is applied to perfonn estimation of nondimensional longitudinal parameters using simulated short period data of an aircraft. The data is simulated with a sampling time of 0.03 second by giving a doublet input to the elevator. Random process noise (zero mean and Gaussian) with standard deviation of 0.001 is added to certain states. The noisy measurements with SNR-10 are generated. For estimating the parameters using DFKF. they are modeled as augmented states in the state model. In this case there are $\mathbf{4}$ states and 11 parameters to be estimated using 7 observables. The initial states and parameters for the DFKF are assumed to be $10 \%$ off from their tnue values. The initial estimation covariance mix is chosen to reflect this uncertainty. The estimated values of the parameters are compared with the true. values of the derivatives in Table 1. The estimates are fairly close to the true values. The convergence of the pitching moment related derivatives: $\mathrm{C}_{\mathrm{m}_{4},}, \mathrm{C}_{\mathrm{m}_{q^{*}},}, \mathrm{C}_{\mathrm{m}_{\mathrm{q}}}, \mathrm{C}_{\mathrm{m}_{5_{\mathrm{F}}}}$ is shown in Fig. 5. It is clear that even in the presence of noise in the data, the patameters converge close to their true values. However. some deviation is observed for $\mathrm{C}_{\dot{\mathrm{m}}_{\mathrm{o}}}$ estimate.

\section{Conclusions}

The performances of UDEKF and DFKF are compared for applications lie kinematic consistency checking using realistic longitudinal short period and lateral-directional data of an aircraft. It is observed that DFKF performs better than UDEKF and hence can he used for many nun-linear filtering and control applications. Also, a sensitivity study of DFKF was carried out. A data fusion scheme for similar sensors is proposed and its performance evaluated. Application of DFKF is also illustrated for parameter estimation.

\section{References}

[1] Simon J. Julier, Jeffrey K. Uhlmann, A New

Extension ofthe Kalman Filter to Nonlinear Systems,

Proceeding of AeroSense, I I" Imernational Symposium Aerospace/Defense Sensing, Simulation and Controls, 1997, pp. 182-193.

[2] SimonJ. Julier, Jeffrey K. Uhlmann, Unscented Filtering and Nonlinear Estimation, Proceeding of the IEEE, Vol.92, No. 3, March 2004, pp. 40I-422.

[3] Raol J.R, Girija G., Jatinder Singh, Modelling and Parameter Estimation of Dynamic Systems, IEE Control Engineering Series Book, Vol. No. 65, IEE, London, August 2004. 
Table 1 Estimated parameters of an aircraft

\begin{tabular}{|c|c|c|}
\hline Parameter & True values & Estimated \\
\hline$C_{X_{0}}$ & $-\mathbf{0 . 0 5 4 0}$ & $-\mathbf{0 . 0 6 1 6}$ \\
\hline$C_{X_{a}}$ & 0.2330 & 0.2531 \\
\hline$C_{X_{a^{2}}}$ & 3.6089 & 3.6840 \\
\hline$C_{Z_{0}}$ & -0.1200 & -0.1279 \\
\hline$C_{7_{u}}$ & -5.6800 & -5.7084 \\
\hline$C_{Z_{\delta_{e}}}$ & -0.4070 & -0.5033 \\
\hline$C_{m_{o}}$ & 0.0550 & 0.0604 \\
\hline$C_{m_{u}}$ & -0.7290 & -0.7108 \\
\hline$C_{m_{\alpha^{2}}}$ & -1.7150 & -1.7701 \\
\hline$C_{m_{q}}$ & -16.3 & -14.9726 \\
\hline$C_{m_{b_{c}}}$ & -1.9400 & -1.8779 \\
\hline
\end{tabular}

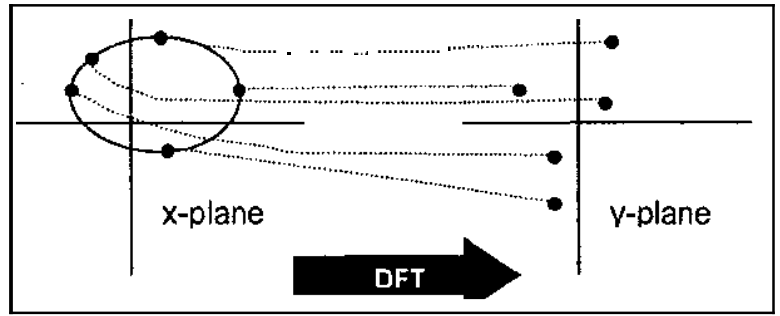

Figure 1. Derivative free transformation
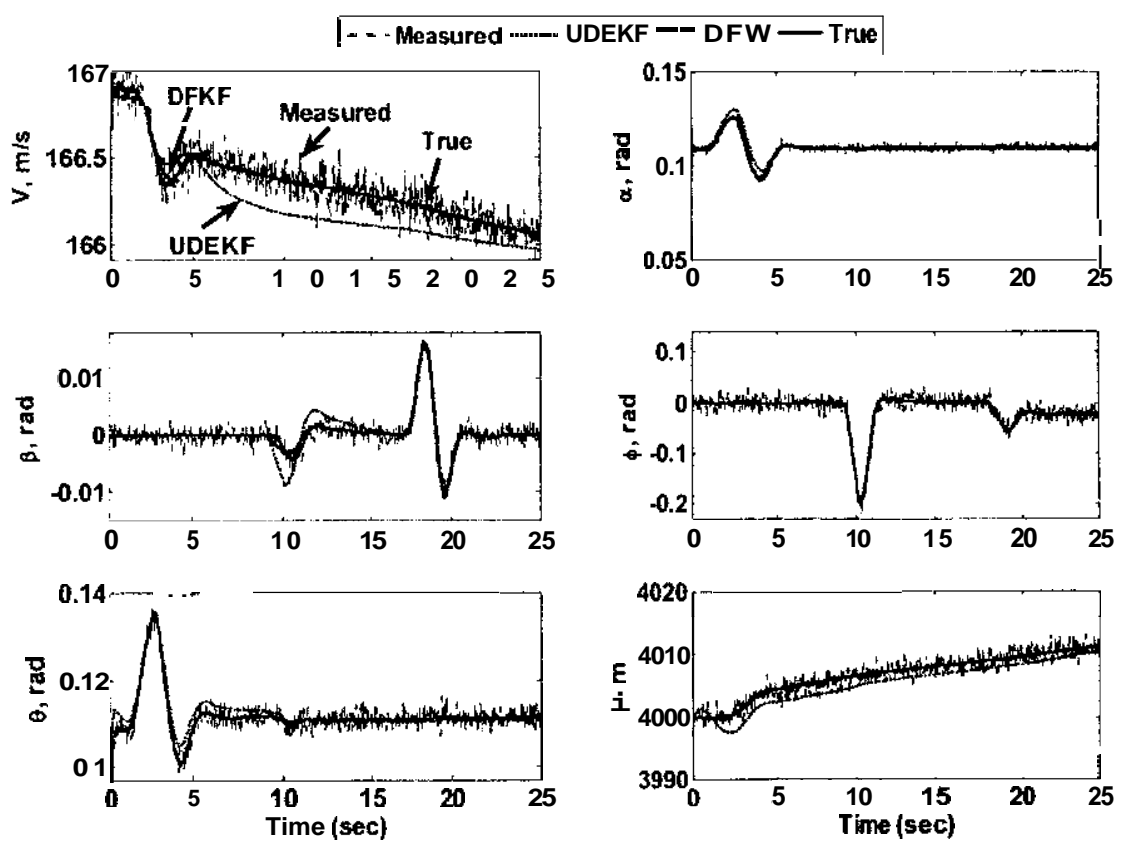

Figure 2. Comparison of true, measured, and estimated obsewation data 


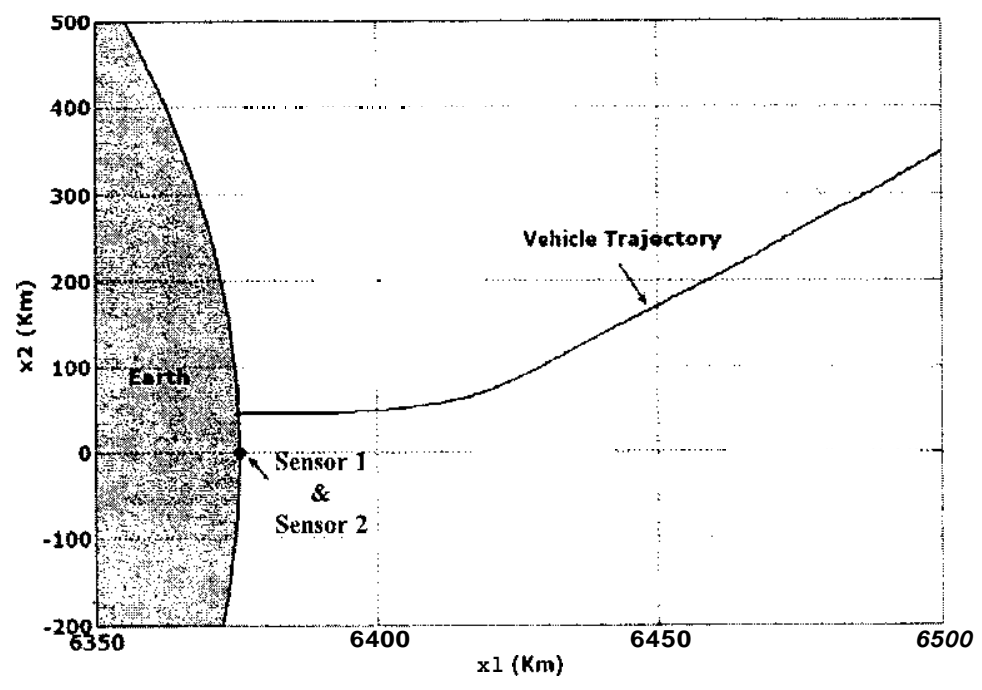

Figure 3. True pasitions of re-entered vehicle tn Earth atmosphere

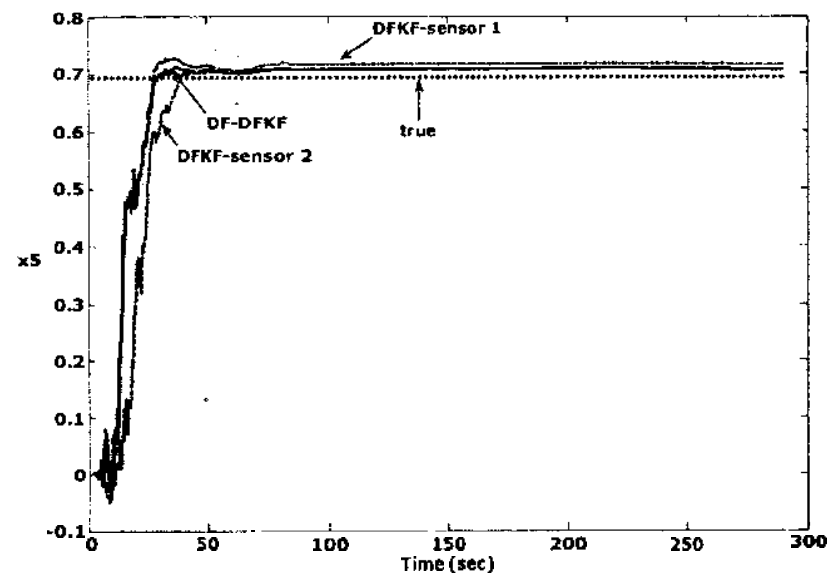

Figure 4. Comparison of true, estimated and fused state $x_{5}$
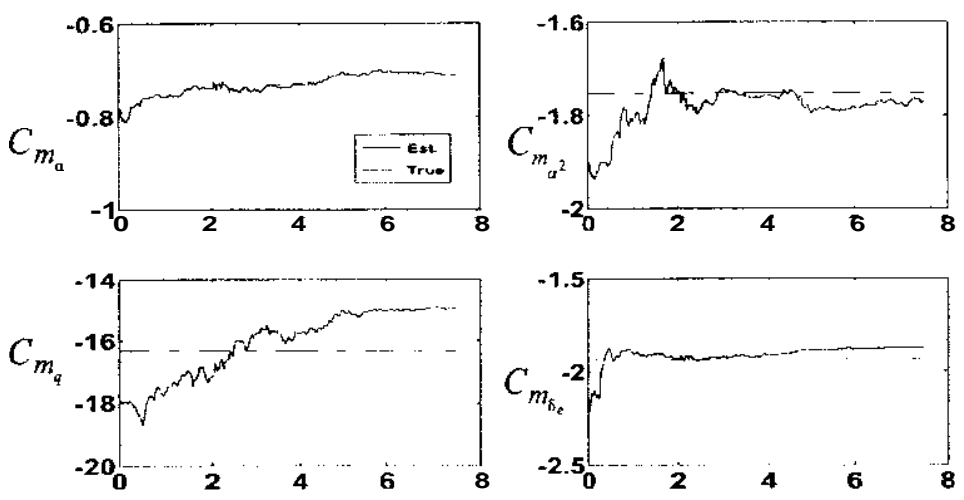

Time (sec)

Figure 5. Parameter convergence for an aircraft-pitchingmoment derivatives 